\title{
Retraction Note: Analysis of stress and strain of surrounding rock in goaf based on GIS
}

\author{
Shuai Wang ${ }^{1} \cdot$ Yao Su${ }^{1} \cdot$ Chao Wang $^{1}$
}

Published online: 13 December 2021

(c) Saudi Society for Geosciences 2021

Retraction Note: Arabian Journal of Geosciences (2021) 14: 1843

https://doi.org/10.1007/s12517-021-08164-2

The Editor-in-Chief and the Publisher have retracted this article because the content of this article is nonsensical. The peer review process was not carried out in accordance with the Publisher's peer review policy. Author Shuai Wang has not responded to correspondence regarding this retraction. The Publisher has not been able to obtain current email addresses for authors Yao Su and Chao Wang.

The original article can be found online at https://doi.org/10.1007/ s12517-021-08164-2.

Shuai Wang

wangshuai@lntu.edu.cn

1 School of Civil Engineering, Liaoning Technical University, Fuxin 123000, China 